\title{
Voyager observations of galactic and anomalous cosmic rays in the helioshealth
}

\author{
F.B. McDonald ${ }^{1}$, W.R. Webber ${ }^{2}$, E.C. Stone ${ }^{3}$, A.C. Cummings ${ }^{3}$, B.C. \\ Heikkila $^{4}$ and N. Lal ${ }^{4}$ \\ ${ }^{1}$ Institute for Physical Science and Technology, University of Maryland, College Park, MD, USA \\ ${ }^{2}$ Department of Physics and Astronomy, New Mexico State University, Las Cruces, New Mexico, USA \\ ${ }^{3}$ California Institute of Technology, Pasadena, CA, USA \\ ${ }^{4}$ NASA/Goddard Space Flight Center, Greenbelt, MD, USA
}

\begin{abstract}
Anomalous cosmic rays display large temporal variations at the time and location where Voyager 1 (V1) crossed the heliospheric termination shock $(2004.86)\left(94 \mathrm{AU}, 34^{\circ} \mathrm{N}\right)$. On a short time scale (3 months) there was a large decrease produced by a series of merged interaction regions (MIR), the first of which was associated with the intense Oct./Nov. 2003 solar events. On a longer time scale there is a remarkable correlation between changes in the galactic cosmic ray (GCR) intensity and those of $10-56 \mathrm{MeV} / \mathrm{n}$ ACR He and 30-56 MeV H extending over a 4.3 year period with the GCRs exhibiting their expected behavior over this part of the 11 and 22 year solar activity and heliomagnetic cycle. The relative changes in the ACR and GCR are the same for both the short term and long term variations. The comparative V1/V2 ACR and GCR spectra in the foreshock and heliosheath indicate that at this time most of the higher energy ACRs are not being accelerated near V1 but must have their source region elsewhere - possibly near the equatorial region of the TS as was suggested in our first paper on the TS crossing (1).
\end{abstract}

Keywords: Galactic Cosmic Rays, Anomalous Cosmic Rays, Termination Shock, Heliosheath, Voyager

PACS: $96.50 . \mathrm{S}-$, 96.50.Ek, $98.70 . \mathrm{Sa}$

\section{INTRODUCTION}

On December 16, 2004 at 94AU V1 crossed the heliospheric termination shock (TS) and entered the region of the heliosheath ${ }^{1,2,3}$ where it has remained for the last 16 months. At the TS the ACR He intensity $>\sim 5 \mathrm{MeV} / \mathrm{n}$ was well below the predicted level and along with galactic cosmic ray ions and electrons was significantly below that observed at the time of the first V1 termination shock particles (TSP) event which started in 2002.54 (85AU).

At the termination shock crossing (TSX) it was expected that:

(a) Since the TS was the most probable source of the ACRs then the ACR source spectrum should be observed ${ }^{4,5}$.

(b) As was first pointed out by Jokipii et al. ${ }^{6}$, GCRs should experience modest reacceleration as they traverse the TS $7,8,9$

(c) The TS should play a role in further accelerating the low energy solar/interplanetary ions associated with the merged interaction regions (MIRs) that sweep across it.

However, these energetic particle populations did not peak at the TS and continued to increase as V1 moved into the heliosheath

CP 858, Physics of the Inner Heliosheath,

edited by J. Heerikhuisen, V. Florinski, G. P. Zank, and N. V. Pogorelov

(C) 2006 American Institute of Physics 978-0-7354-0355-0/06/\$23.00 
At the TSX there was a large increase in the magnetic field strength ${ }^{1}$ and in the intensity of ions $<0.5 \mathrm{MeV}$, consistent with theoretical expectations. At the same time the large field aligned flow and the frequent temporal variations of the TSP events essentially vanished ${ }^{1,2,3}$.

TSP, ACR and GCR intensities at V1 in late 2004 were at a low level due to the passage of a series of interplanetary disturbances over the previous several months ${ }^{10}$. After the passage of these transients there began an increase in all 3 components at 2004.87, some 30 days before the TSX, that has continued up to the present time. Over this period there has been a close correlation between the ACR H and He, and GCR recovery rates.

These GCR and ACR intensity increases are also present in the V2 data, suggesting that the increases are dominated by temporal and not spatial effects. The first V2 TSP event begins at 2004.87, following the passage of the strong transients beyond the TS and consistent with the onset of the V1 recovery.

In mid-2005 the GCR recovery at $1 \mathrm{AU}$ (as defined by $265 \mathrm{MeV} / \mathrm{n}$ GCR He) from Solar Maximum to the expected Solar Minimum condition was $\sim 55 \%$ complete ${ }^{11}$. From the period just prior to the late October, early November 2003 series of intense solar events to 2006.25, there has been a close correlation between changes in the 1AU and Voyager 2 (V2) GCR intensity when a convection correction is applied.

It will be argued that the MIRs produced by the October/November 2003 Halloween events and subsequent solar activity over the ensuing 90 days is the major cause of the low ACR and electron intensity at the TSX. The relative changes in the ACRs and GCR, in both the foreshock and heliosheath regions indicate that longer term temporal variations are present. The TSP events at V1 and V2 provide essential clues for understanding the evolution of the three energetic particle populations in the heliosheath.

\section{OBSERVATIONS}

We define TSP events by the time history of low energy $2.5 \mathrm{MeV} \mathrm{H}$ (Fig.1). The first TSP event started in 2002.5 and persisted for some six months followed in 2003.7 by a second year long increase. As noted previously, these TSP events are highly variable, have relatively flat energy spectra and frequent periods of streaming along the expected direction of the interplanetary magnetic field. The composition of the TSP ions resembles that of ACRs as defined by the high $\mathrm{O} / \mathrm{C}$ ratio ${ }^{10,12,13}$. With their power-law spectra extending below $100 \mathrm{keV}$ and frequent field aligned streaming these TSPs at V1 monitored the energetic particle populations at mid-heliolatitudes of the TS for some 2.4 years before the actual TSX.

In the foreshock region, interplanetary transients in the form of MIRs play a major role in the two V1 TSP events. 


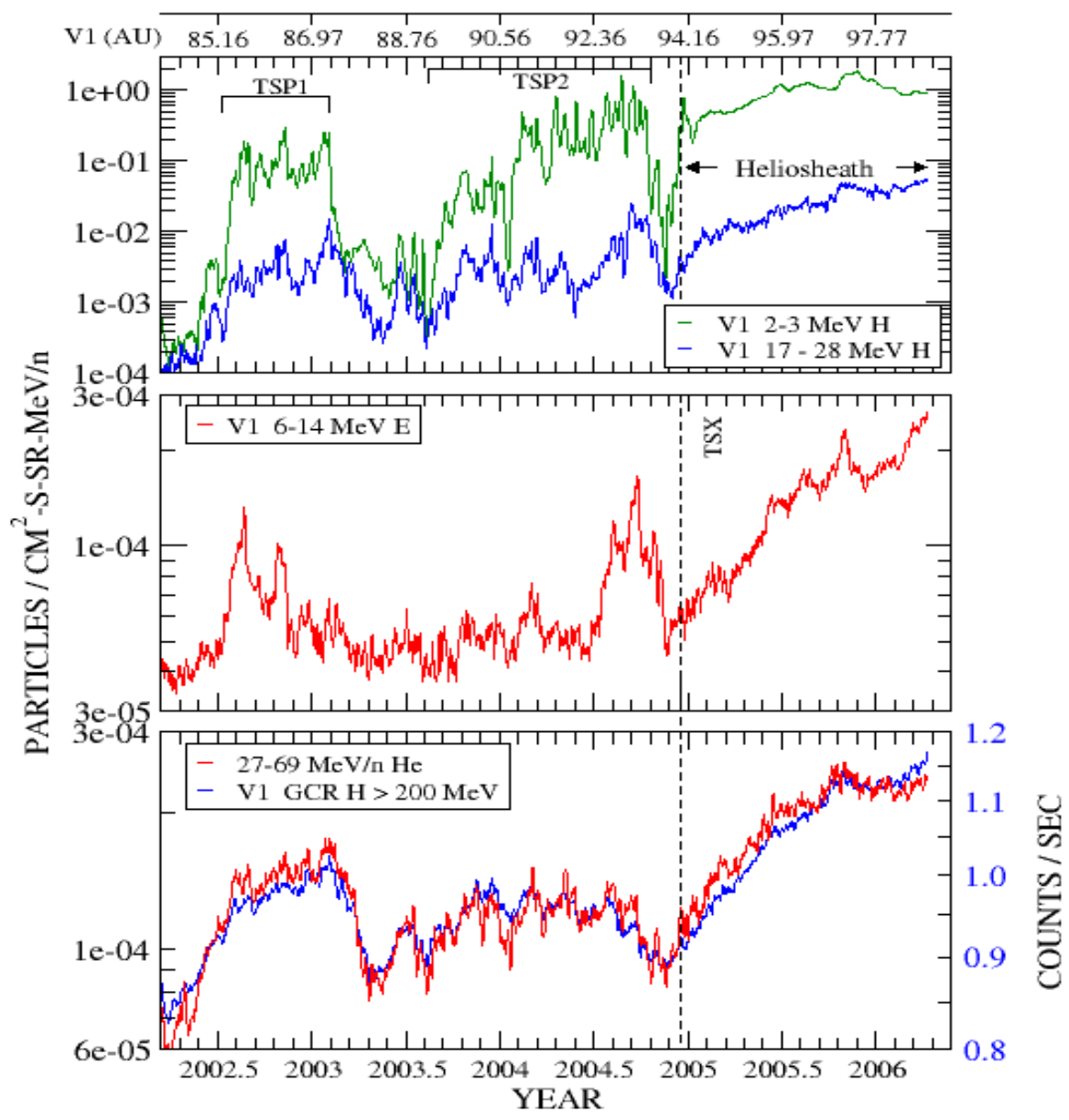

Figure 1. V1 time histories (5 day moving averages) of (a) 2-3 and 17-28 MeV H, (b) 6-14 MeV electrons and (c) 27-69 MeV/n He and the GCR H > $200 \mathrm{MeV}$ rate. The dashed line marks the TSX and the time of the TSPs are shown in the top panel. All data in this and the following figures are from the Voyager Cosmic Ray Subsytem experiment (E.C. Stone, P.I.).

These MIRs also frequently produce an impulsive increase of 2-20 MeV electrons and $\mathrm{H}$ ions extending up to $50 \mathrm{MeV}$ (Fig 1a,b). Since entering the heliosheath there have been transient increases above the steadily rising electron intensity, with the largest such increase peaking at 2005.82 - most probably related to the CMEs and subsequent MIR produced by the large solar event of January 20, 2005 along with other events near that time.

An overview of the relative changes in the ACR (27-69 Mev/n He) intensity and the GCR integral $\mathrm{H}$ intensity ( $>200 \mathrm{MeV}$ ) shows (Fig. 1c) their close correlation that extends over the TSP increases and through the first 16 months in the heliosheath. The 
intensity of both components is larger in TSP 1 than TSP 2. The dip in both components starting around 2005.82 is related to the passage of the MIR producing the electron peak (1b)

Clearly evident from the data in all 3 panels of Fig. 1 is the minimum at 2004.87, some 30 days before the TSX. This decrease, as defined by the $>200 \mathrm{MeV}$ GCR H rate, starts at 2004.62 and consists of a series of step decreases suggesting the passage of more then 1 MIR. The $1^{\text {st }}$ step decrease was associated with the October/November 2003 solar events.

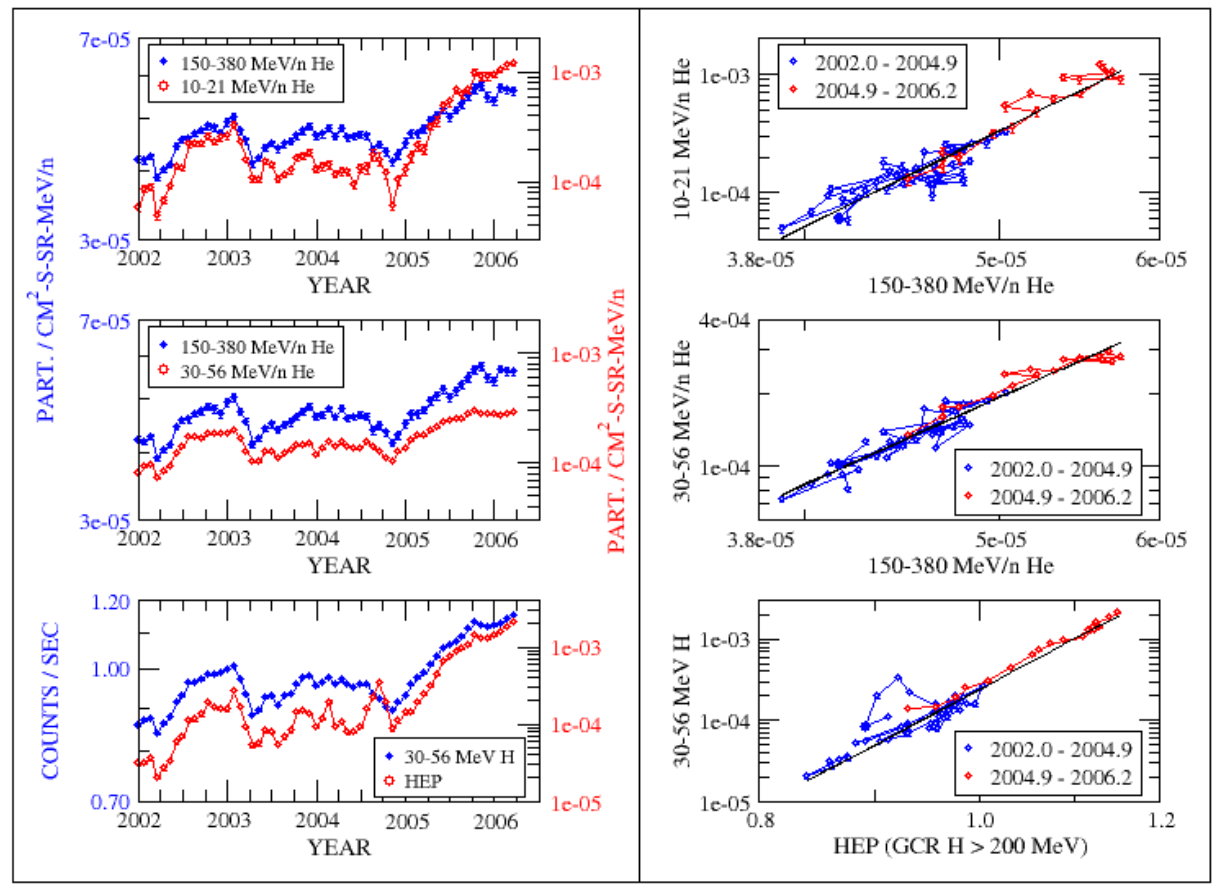

Figure 2. V-1 Cross-correlation plots (26 day averages) of (a) 10-21 MeV/n ACR He vs 150-380 $\mathrm{MeV} / \mathrm{n}$ GCR He, (b) 30-56 MeV/n ACR He vs 150-380 MeV/n GCR He and (c) 30-56 MeV H vs an integral GCR $\mathrm{H}>200 \mathrm{MeV}$. The solid lines are least-squares fit to the data. The excursion that peaks at the HEP value of 0.91 is produced by the passage of several MIRs that are clearly seen in the 6-14 MeV electrons and 17-28 MeV H data from 2004.62 to 2004.8 (Fig. 1). The solid blue data point in each panel is the data period just prior to the TSX. The heliosheath data is shown in red in the three cross-correlation panels. The HEP is an integral rate that responds to $>200 \mathrm{MeV} \mathrm{H}$.

This relation of the relative changes in the GCRs and ACRs can be examined in greater detail with cross-correlation plots over the period 2002.0 - 2006.14 (Fig. 2) using 150 $380 \mathrm{MeV} / \mathrm{n}$ GCR He vs 10-21 MeV/n and 30-56 MeV/n ACR He, and 30-56 MeV H vs > $200 \mathrm{Me} \mathrm{V} \mathrm{H}$. Near the beginning of this period, the 10-21 MeV/n ACR He and 30-56 $\mathrm{MeV} \mathrm{H}$ are at their lowest point in cycle 23 while the higher energy $30-56 \mathrm{MeV} / \mathrm{n} \mathrm{He}$ had already begun its initial recovery. The data after the TSX is shown in red while the minimum value just prior to the TSX is marked by a solid blue data point along with an 
attached arrow. A least squares fit to the data is shown as a solid line. In each case, the ACRs and their GCR counterpart are recovering along the same regression line. The effect of the larger series of MIRs is to move the ACRs and GCRs back down this regression line, and then recover along this same line as V1 moved into the heliosheath in the ongoing recovery phase of cycle 23 .

Similar plots of the V2 ACR He data (not shown) after the onset of the V2 TSP event in 2005.87 follow a regression line that is parallel to that of $\mathrm{V} 1$ but displaced below it while the V2 $30-56 \mathrm{MeV} \mathrm{H}$ traces a regression line close to its V1 counterpart.

The V1 and V2 $\mathrm{H}$ and He energy spectra from just before the TSX and 1.1 years later (Fig. 3) provide additional insight on the evolution of the ACR and TSP populations. The changes in the $\mathrm{H}$ spectra over this period, have been discussed in detail by Cummings et al. (14). The lowest energy protons experience a large increase at the TS $(1,3)$ and by early 2006 display several different spectral slopes with increasing energy. The V1 H spectra is somewhat steeper than that of V2 $(\mathrm{V}=2.4 \mathrm{vs} 1.7$ below $10 \mathrm{MeV})$ in early 2006 (Fig. 3c), consistent with increased modulation at V2 at lower energies.
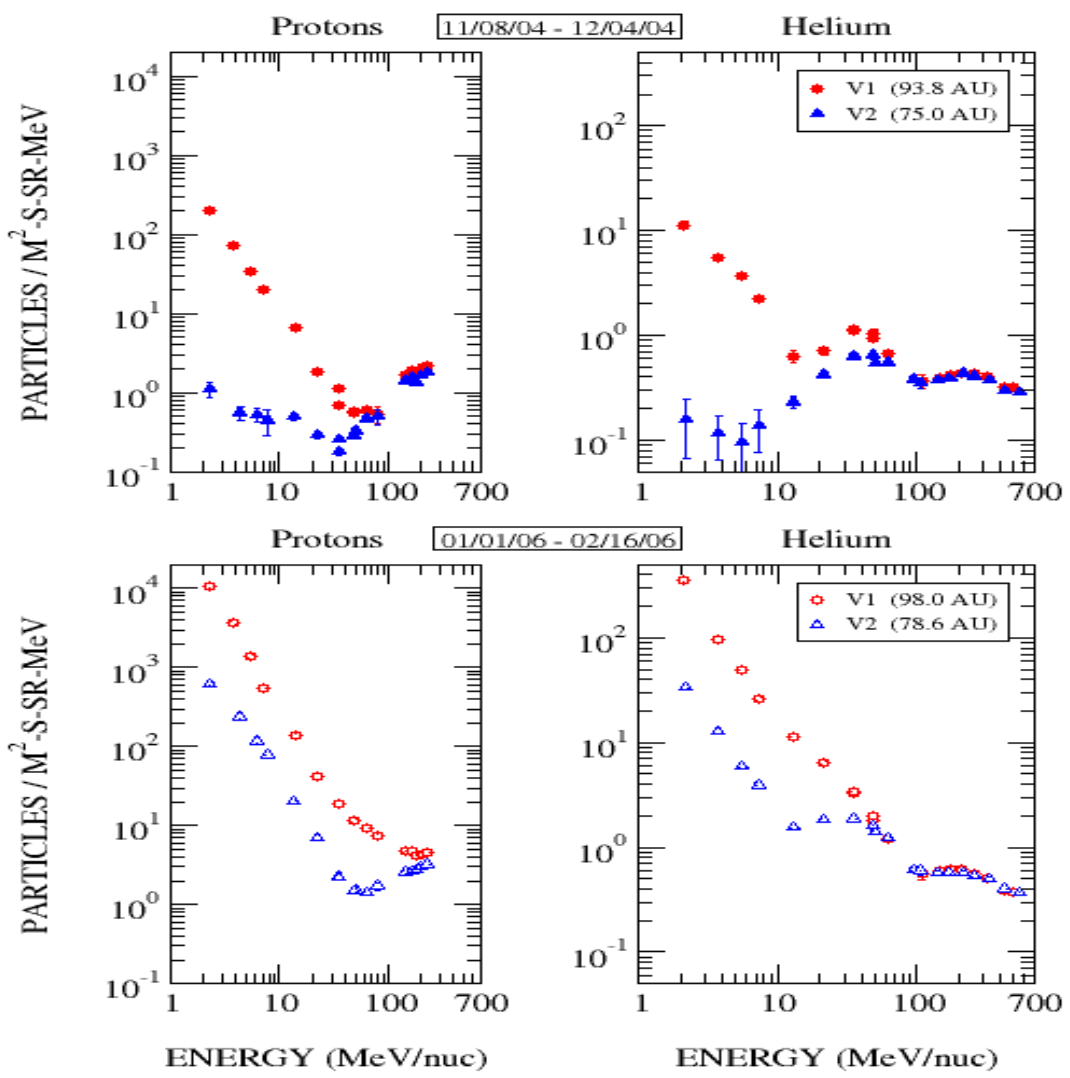

Figure 3. V1 and V2 energy spectra for the period (a) just prior to the V1 TSX and (b) 01/01/2006 $02 / 16 / 2006$ after V1 has been in the heliosheath for 14 months. 
The V2 H and He spectra (10/08 - 12/04/04) show no evidence for TSP or ACR H. However, in early 2006, TSP $\mathrm{H}$ is apparent in the V2 spectrum from 2-40 MeV, although at a reduced intensity compared to the V1 spectrum in the heliosheath. In the foreshock region in late 2004 the V1 GCR, ACR and TSP He are well resolved as in the case for V2 in early 2006, while the V1 He spectra in 2006 has evolved into a double power law that that extends to some $80 \mathrm{MeV} / \mathrm{n}$, spanning most of the ACR region. The ACR He from 42-69 MeV/n and the GCR He $>100 \mathrm{MeV}$ at V1 and V2 are similar despite their 19.4 AU separation. At lower energies ( $<10 \mathrm{MeVnuc})$ the similar spectral slopes of V1 and V2 He confirm that the TSPs are indeed monitors of the energetic particles of the heliosheath in the vicinity of the TS.

\section{DISCUSSION}

The low level of ACRs observed when V1 crossed the TS on 16 December 2004 is mainly the result of two separate processes. The first was a short term transient effect produced by the passage of several strong MIRs starting in 2004.62. The second process is a long term one that appears to be associated with the 11 year cycle of solar activity and possible with the 22 year heliomagnetic cycle. This association is established by the tight correlation between GCRs and the higher energy ACRs (10-56 Mev/n He, 30-56 Mev H) over a 4 year period 2002.0 - 2006.25). Different physical processes may be involved in the ACR and GCR long term temporal variations with each controlled by phenomena associated with some aspect of the long term activity cycles such as CMEs, changes in the magnitude and turbulence of the interplanetary magnetic field and the current sheet inclination. However the higher energy ACRs require acceleration times on the order of more than 6 months and the close correspondence between the long term and short term ACR and GCR temporal variations suggest there could be a common change in the transport process.

At 1AU, from a period just prior to the series of Oct/Nov 2003 solar events to mid 2005, there was a $42 \%$ increase in $265 \mathrm{MeV} / \mathrm{n}$ He. At V2, the increase was 34\%. From Solar Minimum to Solar Maximum (as defined by GCRs), the 1AU this GCR He interval was reduced by a factor of 6 while the corresponding decrease at V2 was 30\% at and $22 \%$ at V1 (11). Previous studies have predicted that the GCR intensity in the outer heliosphere at a given heliocentric distance in the equatorial plane would be greater at a $\mathrm{qA}<0$ solar minimum then for $\mathrm{qA}>0$ based on Pioneer 10, Voyager, and IMP 8 studies

15,16. These observations establish that phenomena originating at the Sun are controlling the modulation in the distant heliosphere and that drifts are important in this region. These drift effects should also influence the ACRs.

The low energy TSP spectra show that these particles can reach the foreshock region ${ }^{12}$. However at V2 the ACR He spectra have a peak at $34 \mathrm{MeV} / \mathrm{n}$ and a distinct minimum before the sharp increase of TSPs at $10 \mathrm{MeV}$. A similar pattern was noted at V1 for TSP 1 and TSP 2 and for the initial period after the TSX. However, the increase of 10-21 $\mathrm{MeV} / \mathrm{n} \mathrm{He}$ is $\sim 5$ times larger than that of $30-56 \mathrm{MeV} / \mathrm{n}$ He over a 4.25 year period. The TSP and ACR He have evolved toward a double power law spectra suggesting that at some time in the recovery phase of cycle 23, it may not be possible to distinguish 
between the two components in the heliosheath. This would be consistent with the ACR like composition of the TSPs ${ }^{(3)}$. However, both the V2 He spectra in early 2006 and that of V1 just after the TSX clearly require that the higher energy ACRs $(>10 \mathrm{MeV})$ are coming from a different location - possibly from the TS near the equatorial region as was suggested in our initial report of the TSX. (1) or from the flanks of the heliosphere (McComas and Schwardron, GRL 33, L04102, 2006) These observations also suggest that the ACR/TSP He source $>5 \mathrm{MeV} / \mathrm{n}$ along the TS varies on a time scale on the order of the 11 year cycle.

\section{REFERENCES}

1. E.C. Stone, Ac. Cummings, F.B. McDonald et al, Science, 309, 2017, 2005

2. L.F. Burlaga, N.F. Ness, M.H. Acuna et al, Science, 309, 2027, 2005

3. R. B. Decker, S.M. Krimigis, E.C. Roelof et al, Science, 309, 2020, 2005

4. M.E. Pesses, J.R. Jokipii and D, Eichler, Astrophys. J., 246, L85, 1081

5. A.C. Cummings, E.C. Stone and C.D. Steenberg,, Astrophys. J., 578, 194, 2002

6. J.R. Jokipii, J Kota and E. Merenyi, Astrophys. J., 405, 782, 1993

7. C.D. Steenberg Ph.D. Thesis, Potchefstroom Univ. Potchefstroom, South Africa

8. R. A. Caballero-Lopez, H. Moraal and F.B. McDonald, J Geophys. Res, 109, A5 (10.1029/2003 JAO 10358) 2004

9. M.S. Potgieter and S.E.S. Ferreira, , J Geophys. Res, 107, A7 (10.1029/2001/Ja U09040), 2002

10. F.B. McDonald, E.C. Stone, L.F. Burlaga, AIP Conf. Proc. 781, 261, 2005

11. F.B. McDonald, B.C. Heikkila, N. Lal and W.R. Webber, Proc. $29^{\text {th }}$ Intl. Cosmic Ray Conf. (SH3.1, 2005)

12. S.M. Krimigis, R.B. Decker, M.E. Hill et al, Nature, 426, 45, 2003

13. A.C. Cumings and E.C. Stone AIP Conf. Proc. 781, 273, 2005

14. A.C. Cummings, E.C. Stone, F.B. McDonald, B.C. Heikkila, N. Lal and W.R. Webber, these proceedings, 2006

15. F.B. McDonald, Space Sci. Rev., 83, 33, 1998

16. W.R. Webber and J.A. Lockwood, J Geophys Res, 106, 29, 323, 2001

17. D.J. McDomas and N.A. Schwadron, Geophys Res Lett, 33, L04102, 2006c 